\title{
Cytoprotective effect of bioactive sea buckthorn extract on paraquat-exposed A549 cells via induction of Nrf2 and its downstream genes
}

\author{
BISWAJIT PODDER, YONG-SIK KIM and HO-YEON SONG \\ Department of Microbiology, College of Medicine, Soonchunhyang University, \\ Cheonan, Chungnam 330-721, Republic of Korea
}

Received March 29, 2013; Accepted October 1, 2013

DOI: $10.3892 / \mathrm{mmr} .2013 .1736$

\begin{abstract}
The extract of sea buckthorn (SBT) [Hippophae rhamnoides L. (Elaeagnaceae)], is used as a food supplement and traditional medicine in numerous countries. This study investigated the protective effects of the functional extract of SBT against paraquat (PQ)-induced toxicity via antioxidant mechanisms in A549 cells. The methanol extract of SBT $(25-200 \mu \mathrm{g} / \mathrm{ml})$ was used to protect cells against PQ $(200 \mu \mathrm{M})$-induced cell death. A viability assay was conducted using 3-(4,5-dimethylthioazol-2-ly)-2,5-diphenyltetrazolium bromide and lactate dehydrogenase (LDH). Total intracellular reactive oxygen species (ROS) were measured and plotted. For validation of the SBT-induced expression of nuclear factor-E2-related factor 2 (Nrf2) and its target genes, western blot analysis and qPCR were performed. The present study showed that pretreatment of A549 cells with SBT extract significantly attenuated PQ $(200 \mu \mathrm{M})$-induced cellular toxicity. The maximum cytoprotective effect was identified using $200 \mu \mathrm{g} / \mathrm{ml} \mathrm{SBT}$ extract; it began $24 \mathrm{~h}$ following exposure and was sustained up to $120 \mathrm{~h}(\mathrm{P}<0.05)$. SBT extract significantly reduced LDH activity by $35.63 \%$ and ROS levels by $30.90 \%$ $(\mathrm{P}<0.05)$. Pretreatment with SBT extract activated $\mathrm{Nrf} 2$ mRNA and protein expression and its nuclear translocation. The SBT extract effectively induced Nrf2 target genes, such as $\mathrm{NAD}(\mathrm{P}) \mathrm{H}$ dehydrogenase quinone 1, glutathione peroxidase 1, glutathione reductase and catalase following treatment with PQ. Based on these results, it was hypothesized that SBT extract may be used as a potential therapeutic agent for the treatment of various oxidative stress-related diseases.
\end{abstract}

Correspondence to: Professor Yong-Sik Kim or Professor Ho-Yeon Song, Department of Microbiology, College of Medicine, Soonchunhyang University, 8 Soonchunhyang 2-gil, Cheonan, Chungnam 330-721, Republic of Korea

E-mail: yongsikkim@sch.ac.kr

E-mail: songmic@sch.ac.kr

Key words: phase II enzymes, phytochemicals, reactive oxygen species, antioxidant-related genes, detoxification

\section{Introduction}

Traditional medicines have been used as primary healthcare in numerous countries and up to $80 \%$ of the world population relies on traditional remedies that are based on plant extracts (1). Plant-derived products, termed phytochemicals, have been extensively investigated and are being used as drugs or dietary supplements due to their availability, the ability for multiple applications, high efficacies and safety (2). The clinical importance of herbal drugs has been on the increase due to the low number of side-effects compared with numerous synthetic antioxidants. Stress management is extremely significant in day-to-day life. The manner in which the body adapts to stress may be supported by food supplements, dietary elements, herbs and minerals, which enhance the immune system. Such compounds are often termed 'adaptogens' (3). It is well known that various plants or plant-derived products are composed of multiple nutrients and antioxidants, including vitamins, flavonoids and polyphenol compounds, and these compounds exhibit direct effects on various diseases, such as cancer and pulmonary diseases (4).

Sea buckthorn (SBT) [Hippophae rhamnoides L. (Elaeagnaceae)] is a thorny nitrogen-fixing arborary shrub, found in numerous countries, including those in Europe and Asia and is a rich source of nutrients (5). SBT has been used as a traditional medicine for thousands of years and is widely used as a therapeutic agent in various human diseases, such as flu, cardiovascular diseases, mucosal injuries, skin disorders, hepatotoxicity and hypoglycemia $(6,7)$. However, the mechanism underlying the involvement of SBT in these diseases has not yet been well characterized. Studies have shown that SBT is a source of nutrients, including vitamins (A, C, E and $\mathrm{K}$, riboflavin and folic acid), carotenoids ( $\alpha$ and $\beta$ carotene and lycopene), organic acids (malic acid and organic acid), tocopherol, lipids, amino acids, tannins and various minerals (7). SBT is also composed of multiple functional ingredients, including sterols (ergosterol, stigmasterol, lanosterol and amyrins) and flavonoids (isorhamnetin, quercetin, myricetin, kaempferol and their glucoside compounds) (7). The predominant chemical constituents isolated and characterized from SBT are antioxidants. Previous studies have investigated the involvement of flavonoids isolated from SBT in protection against 
varioustoxins (8-11). Those flavonoids may also be responsible for its beneficial involvement in paraquat (PQ)-exposed A549 lung cells. Similarly, one of the most important protective mechanisms of flavonoids is their ability to decrease reactive oxygen species (ROS) formation through the activation of Nrf2-dependent genes (8). Notably, the SBT extract has been associated with the induction of detoxifying and antioxidant enzymes as superoxide dismutase (SOD), catalase and glutathione (GSH) (10). Pharmacological and clinical studies have also demonstrated that several flavonoids, which are the active constituents of SBT extract, produce significant antitumor and anti-apoptotic effects $(10,12)$. Plant extracts containing numerous classes of chemical entities with synergistic properties may exhibit greater therapeutic benefits and applicability in lung protection compared with single chemical entities. However, the efficacy of SBT in protection from PQ-poisoning has not yet been elucidated.

PQ (1,1'-dimethyl-44'-bipyridinium chloride) is widely used as a herbicide and has been of great concern to public healthcare due to high morbidity and fatality rates resulting from accidental ingestion or self-poisoning. It is well documented that the toxicity of PQ is based on the generation of excess quantities of ROS, which leads to fatal cellular toxicity in various organs, such as the lung, kidney and liver. PQ toxicity is associated with redox cyclic oxygen and other free radicals, leading to NADPH depletion and the lipid peroxidation of cell membranes $(13,14)$. The lung is the primary target organ of PQ-poisoning and PQ-induced lung toxicity results in the initial development of pulmonary edema, infiltration of inflammatory cells and damage to the alveolar epithelium, which may progress to severe fibrosis (15). The combinatory procedures, i.e., haemoperfusion with antioxidants (vitamin) and/or immune suppressors are used for the treatment of PQ-poisoning; however, these treatments are not highly effective. The use of natural antioxidants may therefore be a promising treatment option for poisoned patients and the effectiveness of such treatments has been previously demonstrated (13). The antioxidant activity or free radical scavenging activity is important in protection against PQ-induced pulmonary toxicity (7). PQ has been shown to result in A549 cell death, which leads to subsequent abnormalities (16).

In the present study, it was hypothesized that SBT extract ameliorates cytotoxicity in PQ-exposed A549 cells by inducing the expression of $\mathrm{Nrf} 2$ and antioxidant-related genes. The results demonstrated that SBT was able to protect cell morphology, membrane integrity and reduce intracellular ROS levels in PQ-exposed A549 cells. Furthermore, SBT induced the activation and expression of $\mathrm{Nrf} 2$ and several detoxifying phase II enzymes, such as $\mathrm{NAD}(\mathrm{P}) \mathrm{H}$ dehydrogenase quinone 1 (NQO1), glutathione peroxidase 1 (GPX1), glutathione reductase $(G S R)$ and catalase $(C A T)$ under the PQ-poisoning condition. The results of this study suggests that SBT is likely a therapeutic candidate for the treatment of PQ-related toxicity.

\section{Materials and methods}

Cells and sample treatment. A549, a human carcinoma cell line (no. CCL-185), was purchased from the American Type Culture Collection (Manassas, VA, USA) and cultured in Dulbecco's modified Eagle's medium (DME) and Ham's F-12
Nutrient mixture culture medium (1:1 mixture; Invitrogen Life Technologies, Carlsbad, CA, USA) containing 10\% heat-inactivated fetal bovine serum and $1 \%$ penicillin/streptomycin. All the experiments were performed on cells maintained within 15 passages. A549 cells have been used in various PQ-induced lung toxicity studies (17). The cells were grown to a confluency of $70-80 \%$ prior to use in the experiments. Cells were treated with different concentrations of SBT and PQ was then added to the culture medium.

Chemicals and reagents. PQ (1,1'-dimethyl-4,4'-bipyridinium dichloride), 3-(4,5-dimethylthiazol-2-yl)-2,5-diphenyltetrazolium bromide (MTT), trypan blue stain solution, and Triton X-100 were obtained from Sigma-Aldrich (St. Louis, MO, USA). DMEM/F-12 was purchased from Invitrogen Life Technologies. Antibiotics/antimycotics $100 \mathrm{U} / \mathrm{ml}$ penicillin, $100 \mu \mathrm{g} / \mathrm{ml}$ streptomycin and $0.25 \mu \mathrm{g} / \mathrm{ml}$ amphotericin B were obtained from Gibco-BRL (Carlsbad, CA, USA) and fetal bovine serum was obtained from HyClone (Thermo Scientific, Rockford, IL, USA). The total ROS detection kit and lactate dehydrogenase (LDH) assay kit were obtained from Enzo Life Sciences (Farmingdale, NY, USA) and Roche (Pleasanton, CA, USA), respectively. The anti-Nrf2 antibody and all secondary antibodies were obtained from Santa Cruz Biotechnology, Inc. (Santa Cruz, CA, USA). The primary antibody against $\beta$-actin was obtained from Abcam (Cambridge, MA, USA). Primers were purchased from Bioneer Corp. (Daejeon, Korea).

Standardized extracts of SBT. A standardized methanol-extract of SBT was purchased from the International Biological Material Research Center in the Korea Research Institute of Bioscience and Biotechnology (Daejeon, Korea). A detailed description of the composition of the SBT extract was described previously by Kim et al (7). Stock solutions $(100 \mathrm{mg} / \mathrm{ml})$ of the extract were prepared in dimethyl sulfoxide (DMSO) and stored at $-20^{\circ} \mathrm{C}$ prior to use.

Cell viability assay. MTT was used as an indicator of cell viability as determined by its mitochondrial-dependent reduction to formazone. Briefly, $2 \times 10^{5}$ cells $/ \mathrm{ml}$ were seeded in a 96-well plate containing a final volume of $200 \mu \mathrm{l}$ and incubated for 24 h to allow the cells to reach a confluency of $80 \%$. The cells were pretreated with different concentrations of SBT for $6 \mathrm{~h}$ prior to $200 \mu \mathrm{M}$ PQ treatment and then incubated for different time periods. Following incubation, $20 \mu \mathrm{l}$ MTT $(5 \mathrm{mg} / \mathrm{ml})$ was added to each well and intercellular reduction of soluble yellow MTT into insoluble purple formazan crystals was allowed to proceed. The supernatant was then removed, and the formazan crystals were dissolved using $100 \mu \mathrm{l}$ DMSO. The plate was then incubated for $30 \mathrm{~min}$ and the optical absorbance of the samples was measured at $590 \mathrm{~nm}$ using a Victor $^{\mathrm{TM}}$ X3 multilabel reader (Perkin Elmer, Waltham, MA, USA). Data on cell viability are shown as the percentage of control (survival of control).

$\mathrm{LDH}$ release assay. $\mathrm{LDH}$ activity assays were performed on A549 cells using a colorimetric technique. Cells were cultured in 96-well plates at $1 \times 10^{5}$ cells/well and grown to a confluence of $70-80 \%$. The cells were then treated with SBT $(25,50$, 100 and $200 \mu \mathrm{g} / \mathrm{ml})$ and PQ $(200 \mu \mathrm{M})$. Following treatment, 
the LDH cytotoxicity assay was performed according to the manufacturer's instructions. Briefly, $100 \mu \mathrm{l}$ cell culture supernatant was transferred from each well to a 96-well plate and $100 \mu \mathrm{l}$ freshly prepared reaction mixture was added to each well. Following $30 \mathrm{~min}$ of incubation at room temperature in the dark, the absorbance was determined at $490 \mathrm{~nm}$ using a Victor X3 multilabel reader (Perkin Elmer). The quantity of LDH was expressed as a percentage compared with the total quantity of LDH present in cells treated with $2 \%$ Triton X-100.

Measurement of total ROS level. Total ROS accumulation in A549 cells was monitored using a fluorescence-based ROS detection kit (Enzo Life Sciences). The experiment was performed according to the manufacturer's instructions.

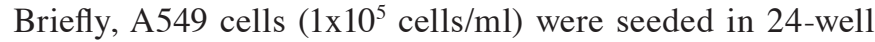
plates and incubated for $24 \mathrm{~h}$. Following incubation, the media were removed from the cell culture plate and the ROS detection solution was added, covering the whole cell surface area. The ROS detection solution was incubated for $1 \mathrm{~h}$ at $37^{\circ} \mathrm{C}$. The cells were then pretreated with SBT for $6 \mathrm{~h}$ and oxidative stress was induced by the addition of PQ $(200 \mu \mathrm{M})$ for $6 \mathrm{~h}$. Pyocyanin and $\mathrm{N}$-acetyl-L-cysteine (NAC) served as positive and negative controls, respectively. Following treatment, the cells were washed with $1 \mathrm{X}$ wash buffer twice to remove all media from the wells. The fluorescence intensity was then measured using a Zeiss Axiovert-25 microscope (Carl Zeiss, Thornwood, NY, USA) with a filter range of Ex/Em: 490/525 nm. The average quantitative value was determined by measuring five different areas of the same sample. The intensity of the fluorescence was proportional to the quantity of total ROS produced by the cells.

Cytoplasmic and nuclear protein separation. Cytoplasmic and nuclear fractions were separated using a commercially available nuclear and cytoplasmic extraction reagent kit (BioVision, Milpitas, CA, USA). A549 cells were seeded in 6-cm dishes at a density of $4 \times 10^{5}$ cells $/ \mathrm{ml}$ and incubated with SBT for $6 \mathrm{~h}$. Oxidative stress was induced by incubation with $200 \mu \mathrm{M} P Q$ for $12 \mathrm{~h}$. Following incubation, the cells were collected, and $0.2 \mathrm{ml}$ cytosol extraction buffer (CEB)-A containing DTT and protease inhibitor were added. Ice-cold CEB B $(11 \mu \mathrm{l})$ was then added and incubated for $10 \mathrm{~min}$. The supernatant containing the cytoplasmic proteins was collected. The remaining pellet was then re-suspended with ice-cold nuclear extraction buffer mix and vigorously mixed for $15 \mathrm{sec}$. Nuclear protein containing supernatant was collected by further centrifugation.

Total protein extraction and western blot analysis. For total cell extracts, A549 cells were grown in 6-well dishes at a density of $4 \times 10^{5}$ cells $/ \mathrm{ml}$ for $24 \mathrm{~h}$ and then treated with the different concentrations of SBT $(25,50,100$ and $200 \mu \mathrm{g} / \mathrm{ml})$ and PQ (200 $\mu \mathrm{M})$. Following treatment, the cells were washed twice with cold phosphate-buffered saline and total cell lysates were prepared using RIPA lysis buffer (Santa Cruz Biotechnology, Inc., Santa Cruz, CA, USA) containing a protease inhibitor cocktail (Santa Cruz Biotechnology, Inc.). The supernatant was collected and the protein concentration was determined using the bicinchoninic acid protein assay kit (Thermo Scientific, Pittsburgh, PA, USA) with bovine serum albumin as a standard control. Proteins $(30 \mu \mathrm{g})$ were separated using a 5\% SDS-PAGE gel at $100 \mathrm{~V}$ for $90 \mathrm{~min}$ and then transferred onto a polyvi- nylidene fluoride membrane (Trans-Blot SD Semi-Dry Cell, Bio-Rad, Hercules, CA, USA) at $15 \mathrm{~V}$ for $1 \mathrm{~h}$. The membranes were blocked by incubating for $1 \mathrm{~h}$ at room temperature with $5 \%$ dried skimmed milk in Tris-buffered saline containing $0.1 \%$ Tween-20. The membranes were then incubated with primary antibodies against $\mathrm{Nrf} 2(1: 500)$ and $\beta$-actin $(1: 10,000)$ overnight at $4^{\circ} \mathrm{C}$. Horseradish peroxidase-conjugated anti-rabbit IgG (Santa Cruz Biotechnology, Inc.) and anti-mouse IgG (Santa Cruz Biotechnology, Inc.) were used as secondary antibodies for Nrf 2 and $\beta$-actin, respectively. Chemiluminescence was performed using an enchanced chemiluminescence system and images were captured using a ChemiDoc Imaging system $\left(\right.$ ChemiDoc $^{\mathrm{TM}}$ XRS+ System with Image Lab ${ }^{\mathrm{TM}}$ Software, Bio-Rad).

qPCR analysis. Total RNA was prepared from A549 cells using an RNA extraction kit (Qiagen, Valencia, CA, USA) according to the manufacturer's instructions. Total RNA was quantified using an ND-1000 spectrophotometer (Thermo Fisher Scientific, Wilmington, DE, USA) at $260 \mathrm{~nm}$ and the purity of the RNA was assessed using the 260/280 nm absorbance ratio. Total RNA $(1 \mu \mathrm{g})$ was used for the generation of cDNA with the Maxime RT PreMix kit (Intron Biotechnology, Korea). qPCR was performed using a CFX96 ${ }^{\text {TM }}$ Real-Time PCR detection system (Bio-Rad) with iQTM SYBR-Green Supermix (Bio-Rad) and gene specific primer sets (for SOD1, GPX1, GSR, CAT, PRDX1 and LPO), which were purchased from Intron Biotechnology (PHS-001050, Accutarget ${ }^{\mathrm{TM}}$ human antioxidant Real-Time PCR primer set, Daejeon, Korea) (17). Gene expression was measured according to the manufacturer's instructions (Bio-Rad). In brief, samples were heated to $95^{\circ} \mathrm{C}$ for $5 \mathrm{~min}$ followed by 40 cycles of $95^{\circ} \mathrm{C}$ for $10 \mathrm{sec}, 42^{\circ} \mathrm{C}$ for $10 \mathrm{sec}, 72^{\circ} \mathrm{C}$ for $20 \mathrm{sec}$ for 40 cycles. Melting curve analysis was performed to ensure the amplification of a single amplicon. Samples were analyzed via the $\Delta \Delta \mathrm{Ct}$ method using GAPDH for normalization and the data are presented as the fold change relative to the control samples. Data were compiled from three independent experiments.

Statistical analysis. Values are expressed as the mean \pm SD. The significance of the difference from the respective controls for each experimental test was analyzed using paired Student's t-test. $\mathrm{P}<0.05$ was considered to indicate a statistically significant difference.

\section{Results}

Protection of cell viability. The MTT assay was performed to investigate the protective effect of SBT in A549 cells exposed to PQ. This cell viability analysis was assessed in a dose- and time-dependent manner with PQ and/or SBT treatment. Pretreatment with the SBT extract was conducted instead of co-treatment with PQ due to its efficacy for cytoprotection(data not shown). As shown in Fig. 1, an SBT extract concentration of up to $200 \mu \mathrm{g} / \mathrm{ml}$ did not result in any cytotoxic effects (Fig. 1A). However, pretreatment with the SBT extract produced a significant cytoprotective effect in cells exposed to PQ (Fig. 1B), whereas PQ treatment alone led to a marked time-dependent reduction in cell viability. Notably, higher concentrations of SBT (100 and $200 \mu \mathrm{g} / \mathrm{ml})$ produced 
A

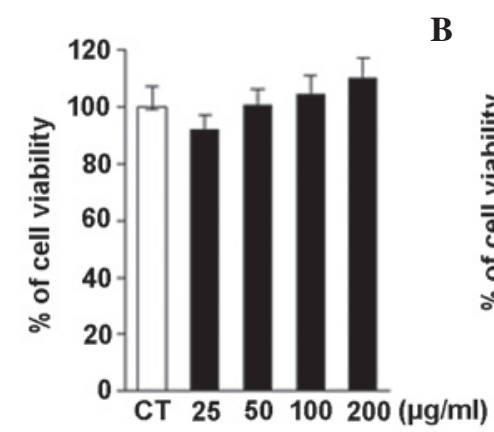

B

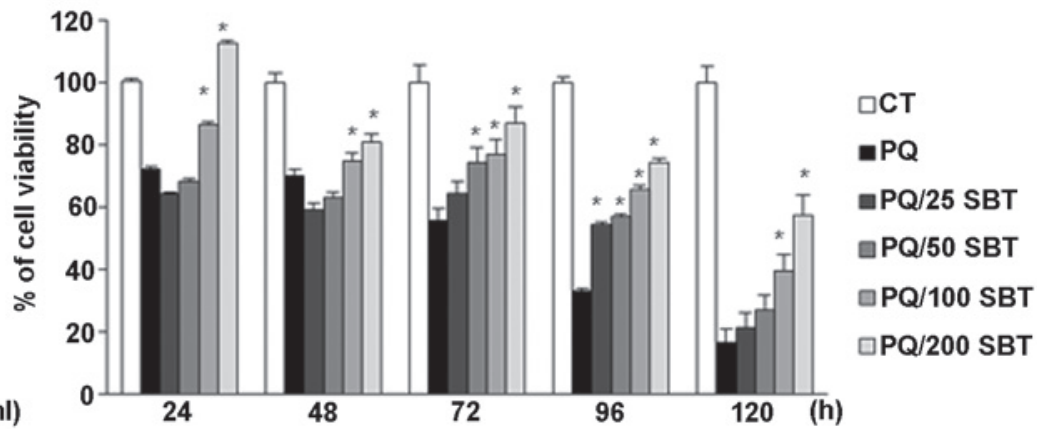

C
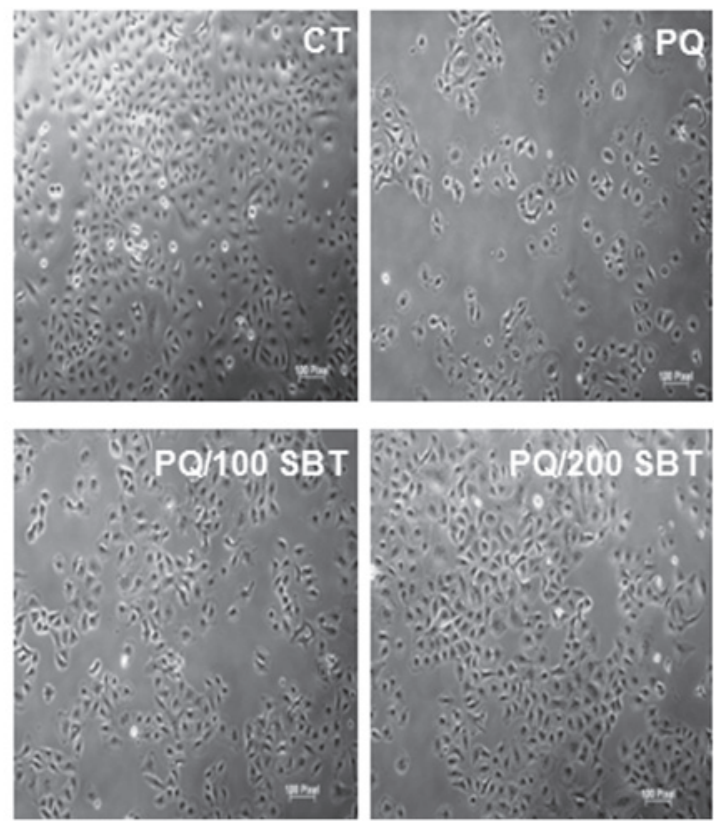

Figure 1. The cytoprotective effects of the SBT extract on PQ-exposed A549 cells. (A) Cells were treated with various concentrations of the SBT extract for $6 \mathrm{~h}$ prior to the addition of $200 \mu \mathrm{M}$ PQ. Cell survival was analyzed using an MTT assay. Additionally, the cells were treated with various concentrations of the SBT extract for $24 \mathrm{~h}$ and cell viability was measured by MTT analysis. (B) Cells were treated with the indicated concentrations of the SBT extract prior to treatment with $200 \mu \mathrm{M}$ PQ. Cell viability was measured at various time points (24, 48, 72, 96 and $120 \mathrm{~h}$ ) as indicated. (C) Morphological studies were performed based on microscopic observations. Images were captured at an original magnification of x10. Results are expressed as a percentage of controls (taken as $100 \%$ ). Data are presented as the mean \pm SEM of at least three separate experiments performed in quadruplicate in each group. "P<0.01 vs. the PQ group. SBT, sea buckthorn; PQ, paraquat; MTT, 3-(4,5-dimethylthiazol-2-yl)-2,5-diphenyl-tetrazolium bromide.

a marked cytoprotective effect at $24 \mathrm{~h}$ and SBT concentrations as low as $25 \mu \mathrm{g} / \mathrm{ml}$ produced a significant cytoprotective effect after $96 \mathrm{~h}$. Moreover, it was observed that this protection effect by SBT was more significant at 72,96 and $120 \mathrm{~h}$. Morphological changes were also observed following PQ treatment. As shown in Fig. 1C, PQ treatment resulted in the cells adopting a round shape, detaching from the surface and aggregating. However, following pretreatment with 100 and $200 \mu \mathrm{g} / \mathrm{ml} \mathrm{SBT}$, A549 cells showed a healthier morphology when compared with PQ-treated cells. Data indicated that SBT demonstrated no cytotoxicity and protected A549 cells against PQ-induced toxicity.

SBT extract preserves membrane integrity. To investigate the effect of the SBT extract on membrane integrity, an LDH enzyme release assay was conducted (Fig. 2). The quantity of released LDH was investigated in a dose- and time-dependent manner. PQ treatment $(200 \mu \mathrm{M})$ led to a $35 \% \mathrm{LDH}$ release; however, the quantity of released LDH was markedly reduced by SBT pretreatment in a dose-dependent manner (Fig. 2A).
$\mathrm{LDH}$ release was low when the cells were pretreated with 100 and $200 \mu \mathrm{g} / \mathrm{ml} \mathrm{SBT}$ following $200 \mu \mathrm{M}$ PQ treatment, which suggested that pretreatment with SBT extract greatly reduces $\mathrm{LDH}$ release induced by $\mathrm{PQ}$ treatment. In addition, the reduction in $\mathrm{LDH}$ release was also shown to be time dependent (Fig. 2B). At 6 h, PQ induced $\sim 40 \%$ LDH release; however, $\mathrm{LDH}$ release was only $25 \%$ when the PQ-treated cells were pretreated with SBT. In addition, the SBT extract alone did not result in increased LDH activity (data not shown), indicating that pretreatment with SBT extract protected the cell membrane integrity against PQ treatment.

Pretreatment of SBT extract decreases total ROS generation. In order to investigate the antioxidant properties of SBT extract, intracellular ROS generation was measured using a total ROS detection kit. Total ROS was analyzed based on the fluorescence intensity from five different regions in three individual experiments. Pyocyanin treatment was used as the positive control and NAC, which is a well-known ROS scavenger, served as the negative control. ROS generation was 

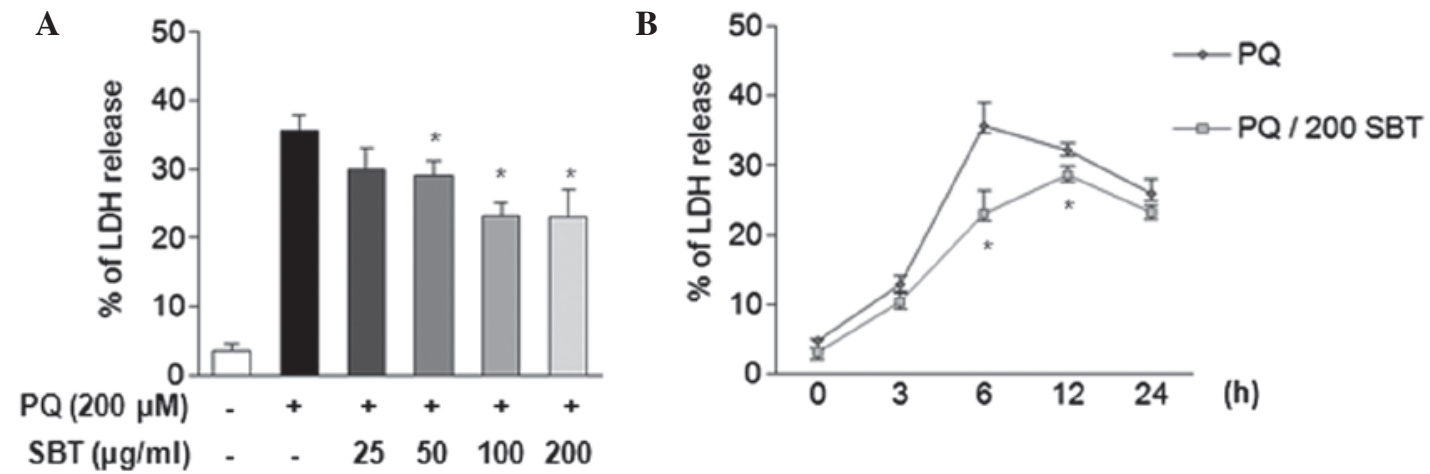

Figure 2. Maintenance of membrane permeability in PQ-exposed A549 cells by SBT extract treatment. LDH activity was measured using the LDH reagent (Roche) in a dose- and time-dependent manner (A and B, respectively). Control cells that were cultured in dimethyl sulfoxide supplemented media and Triton X-100 served as the positive controls and were analyzed in parallel. The data are expressed as the mean $\pm \mathrm{SD}$ of three independent experiments. $\mathrm{P}<0.01$, compared with the control group. PQ, paraquat; SBT, sea buckthorn; LDH, lactate dehydrogenase.

increased by $>60 \%$ following pyocyanin treatment relative to the control cells. In addition, ROS generation was $55 \%$ greater subsequent to PQ treatment when compared with the control cells. Notably, the total intracellular ROS level was markedly reduced in SBT-pretreated A549 cells (Fig. 3). Treatment with $100 \mu \mathrm{g} / \mathrm{ml} \mathrm{SBT}$ extract significantly reduced PQ-induced ROS levels in A549 cells. Moreover, PQ-induced ROS generation was almost completely eliminated with $200 \mu \mathrm{g} / \mathrm{ml}$ SBT extract. In addition, pretreatment with $200 \mu \mathrm{g} / \mathrm{ml} \mathrm{SBT}$ extract reduced ROS generation in pyocyanin-treated cells by $40 \%$. These results suggest that SBT extract was able to function as a ROS scavenger following PQ treatment.

SBT extract activates the antioxidative transcription factor, Nrf2. Nrf2 is pivotal in the host defense mechanism and numerous key phase II enzymes, such as NQO1 and HO1, are controlled by Nrf2 transcription factor under ROS conditions. Therefore, the present study aimed to determine whether SBT extract induces the expression of Nrf2, its downstream target genes and other antioxidant-related genes in PQ-exposed A549 cells. The SBT extract was shown to induce Nrf2 expression (Fig. 4A). A549 cells were treated with different concentrations of the SBT extract for $6 \mathrm{~h}$, total cell extracts were harvested and western blot analysis was then performed. Nrf2 expression was markedly induced by SBT extract treatment (Fig. 4A). Nrf2 mRNA expression was also induced in a dose-dependent manner following SBT extract treatment (Fig. 4B). The effect of SBT treatment on Nrf2 expression under PQ-exposed conditions was also observed (Fig. 4C). PQ marginally induced Nrf2 expression at 6 and $12 \mathrm{~h}$ and the expression of $\mathrm{Nrf} 2$ was significantly induced subsequent to SBT extract pretreatment (Fig. 4C). In order to determine whether the SBT extract affected Nrf2 nuclear translocation, western blot analysis was also performed using fractionized protein samples from PQ and SBT extract-treated cells (Fig. 4D). Treatment with $200 \mu \mathrm{g} / \mathrm{ml}$ SBT extract resulted in the nuclear translocation of Nrf2 in the absence or presence of PQ treatment; however, PQ treatment alone did not result in Nrf2 nuclear translocation. These data suggested that the SBT extract effectively induced the expression of $\mathrm{Nrf} 2 \mathrm{mRNA}$ and activated Nrf2 followed by nuclear translocation.

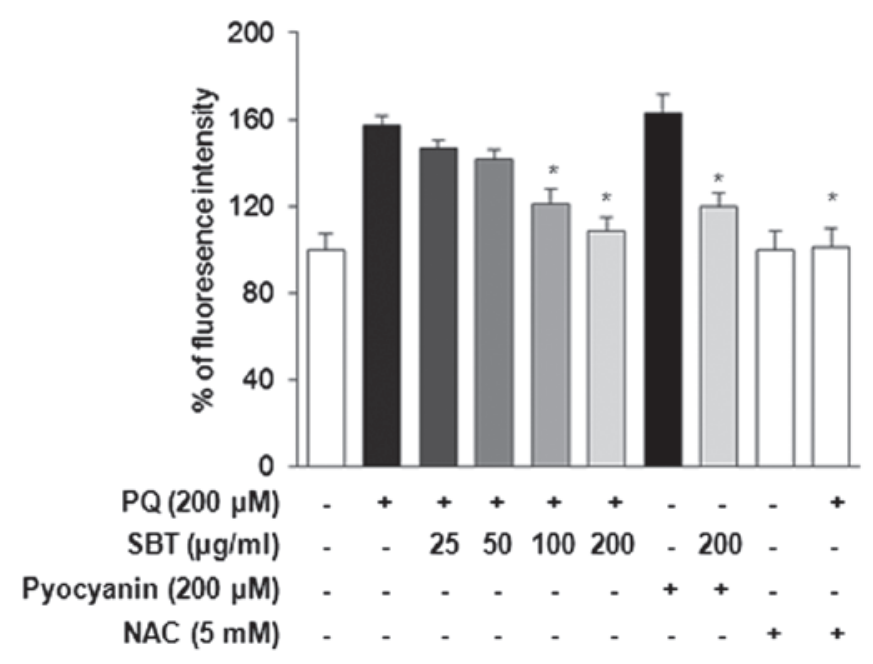

Figure 3. Reduction of intracellular ROS production by SBT extract. A549 cells were loaded with the ROS reagent for $1 \mathrm{~h}$, then treated with $200 \mu \mathrm{M}$ PQ and/ or different concentrations of SBT. As a marker of intracellular ROS levels, quantitative fluorescence was measured by counting five different regions from three individual experiments. Pyocyanin (ROS inducer) and NAC (ROS inhibitor) served as the positive and negative controls, respectively. Bars present the mean \pm SD of three independent experiments. $P<0.01$ vs. the PQ group. ROS, reactive oxygen species; SBT, sea buckthorn; PQ, paraquat; NAC, $N$-acetyl-L-cysteine.

Modulation of antioxidant-related gene expression by SBT treatment. To investigate the effect of SBT extract on the expression of antioxidant-related genes and phase II detoxifying genes, qPCR analysis was performed under various conditions (Fig. 5). Total RNA was collected from PQ-exposed cells at 6 and $12 \mathrm{~h}$ and qPCR was conducted using gene-specific primer sets (Table I). As shown in Fig. 5, PQ alone marginally induced NQOI, GSR and lipid peroxidase ( $L P O)$ gene expression at 6 and $12 \mathrm{~h}$. However, PQ treatment did not effectively induce heme oxygenase 1 (HO1), GPXI, SODI, CAT and peroxiredoxin 1 ( $P R D X 1)$ expression. NQO1, GPXI and GSR mRNA was significantly induced at 6 and $12 \mathrm{~h}$ following SBT treatment, while $H O 1, S O D I$ and PRDXI expression was not altered by SBT treatment. CAT mRNA expression was significantly induced at only $6 \mathrm{~h}$ when PQ-induced A549 cells were exposed to high concentrations $(200 \mu \mathrm{g} / \mathrm{ml})$ of SBT 
Table I. Primers used for qPCR analysis

Gene name

Primer sequence

Amplicon size (bp)

\begin{tabular}{ll}
\hline $\mathrm{Nrf2}$ & Forward: 5'-GCGACGGAAAGAGTATGAC-3' \\
& Reverse: 5'-GTTGGCAGATCCACTGGTTT-3' \\
$\mathrm{NQO1}$ & Forward: 5'-CGCAGACCTTGTGATATTCCAG-3' \\
& Reverse: 5'-CGTTTCTTCCATCCTTCCAGG-3' \\
& Forward: 5'-GCAACCCGACAGCATGC-3' \\
HO1 & Reverse: 5'-TGCGGTCGAGCTCTTCTG-3' \\
& Forward: 5'-TCCCATCACCATCTTCCA-3' \\
& Reverse: 5'-CATCACGCCACAGTTTCC-3' \\
\hline
\end{tabular}

Nrf2, nuclear factor-E2-related factor, $N Q O 1$, NAD(P)H dehydrogenase quinone 1; HO1, heme oxygenase 1; GAPDH, glyceraldehyde 3-phosphate dehydrogenase.

$\mathbf{A}$

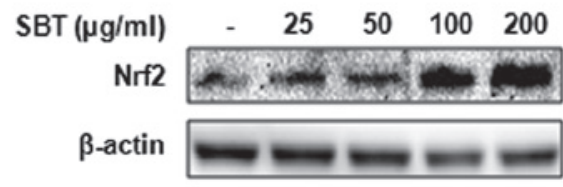

C

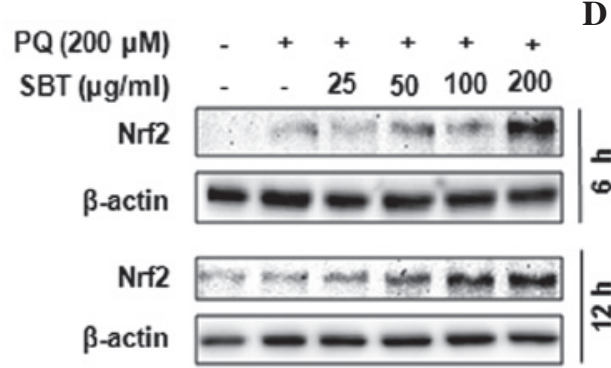

B

D
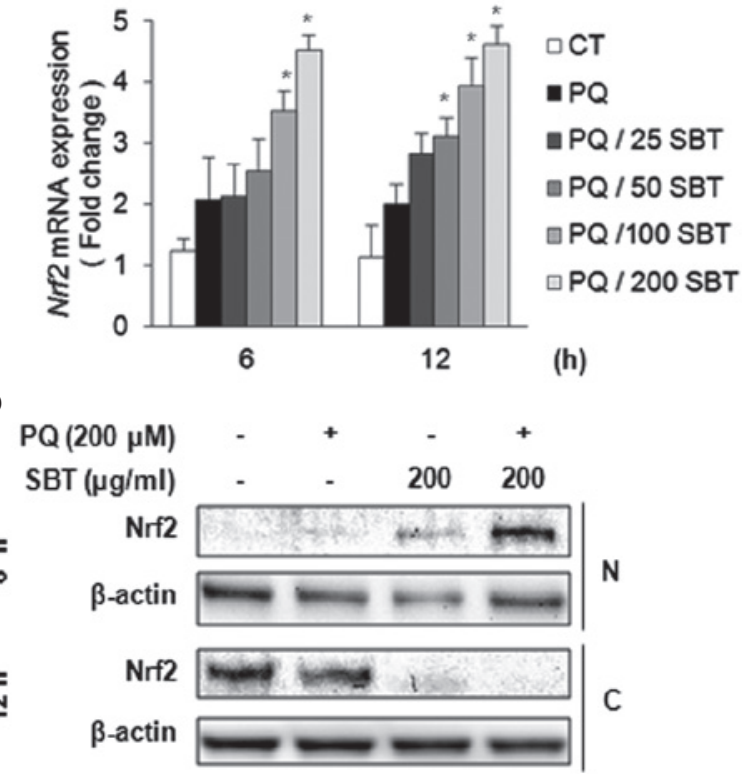

Figure 4. Induction of Nrf2 expression by treatment with SBT extract. (A) A549 cells were pretreated with different doses of SBT (25, 50, $100 \mathrm{and} 200 \mu \mathrm{g} / \mathrm{ml})$. (B) Nrf2 mRNA expression was measured at 6 and $12 \mathrm{~h}$. (C) Nrf2 protein levels were expressed at the different SBT concentrations in A549 cells exposed to PQ for 6 and 12 h. (D) Representative immunoblots of nuclear and cytosolic proteins demonstrating changes in the subcellular localization of Nrf2 following SBT pretreatment in PQ-exposed cells. The data are expressed as the mean $\pm \mathrm{SD}$ of three independent experiments. " $\mathrm{P}<0.05$, compared with the control group. Nrf2, nuclear factor-E2-related factor 2; SBT, sea buckthorn; PQ, paraquat; N, nuclear fraction; C, cytosolic fraction.

extract (Fig. 5F). These results suggested that the SBT extract effectively induced the expression of NQO1, GPX1, GSR and $C A T$ antioxidant-related genes, but not HO1, SOD1, PRDX1 and $L P O$ in PQ-treated A549 cells.

\section{Discussion}

Recent studies have demonstrated that SBT extract contains various functional ingredients, including isoflavonoids, polyphenols and numerous vitamins, and has clinical benefits such as antioxidant effects in various human diseases $(6,10,17-19)$. Therefore, the effect of the SBT extract on ROS-induced cytotoxicity due to PQ-poisoning was investigated. To obtain insights into the involvement of SBT in PQ poisoning, several in vitro experiments were conducted, including an MTT assay,
LDH assay and total ROS measurement, using lung adenocarcinoma A549 cells. A549 cells are a cell line derived from the lung and have been commonly used to investigate the molecular and cellular mechanisms of pulmonary-related diseases (20). In addition, A549 cells have been used extensively to study the mechanisms underlying PQ-induced cell death $(16,20)$. Furthermore, the optimal conditions of SBT treatment were determined. In these experiments, pretreatment with SBT was shown to produce a greater outcome than PQ co-treatment with regard to cytoprotection (data not shown). Thus it was speculated that pretreatment rapidly activates the expression of early host defense genes.

According to the MTT analysis, SBT extract significantly protected A549 cells against death induced by exposure to high concentrations of PQ (Fig. 1). A similar cytoprotective 


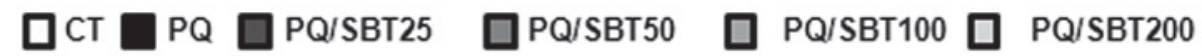

A

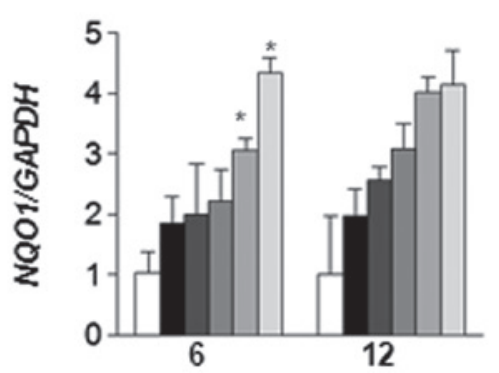

C

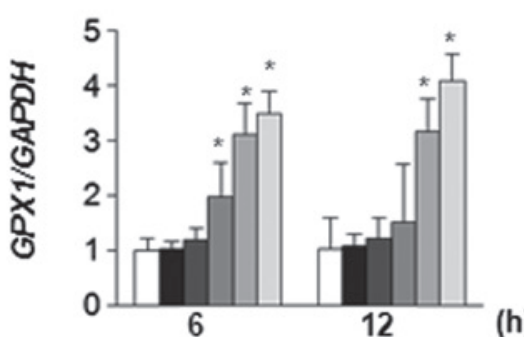

E

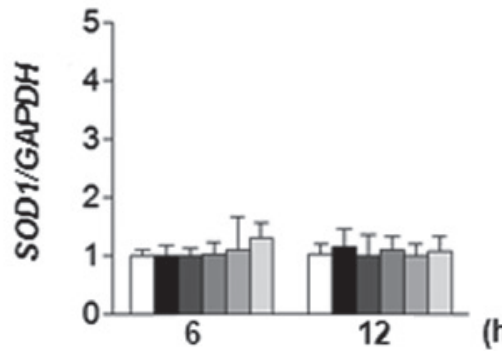

G

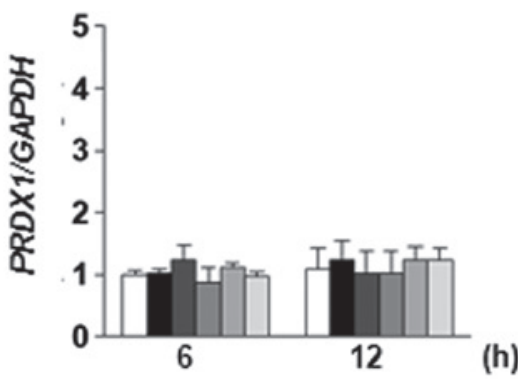

B

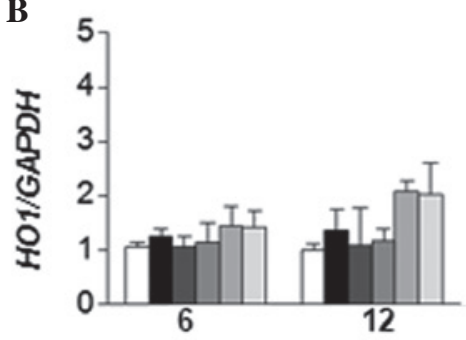

(h)

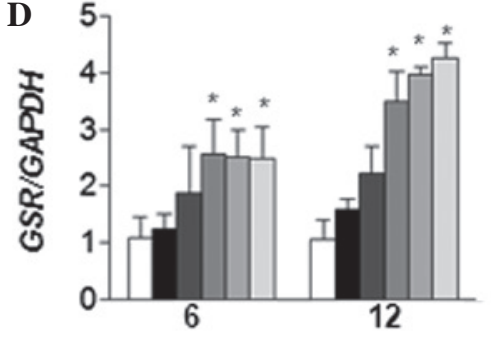

(h)
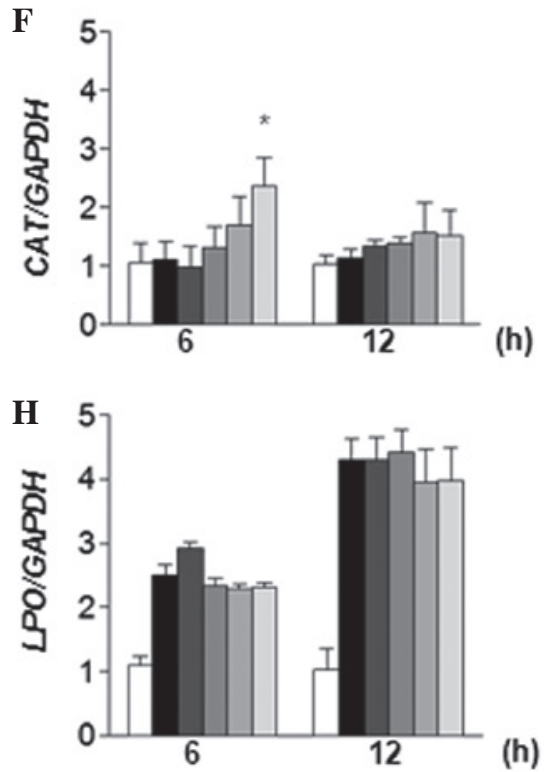

Figure 5. Differentially regulated expression of antioxidant-related genes by SBT (25, 50, 100 and $200 \mu \mathrm{g} / \mathrm{ml}) \mathrm{in} \mathrm{PQ}-\mathrm{exposed} \mathrm{A} 549$ cells. (A) NQO1, (B) $H O 1$, (C) GPX1, (D) GSR, (E) SOD1, (F) CAT, (G) PRDX1 and (H) LPO. The value was monitored using SYBR-Green dye and normalized by glyceraldehyde 3 -phosphate dehydrogenase quantitative measurements. The data are expressed as the mean $\pm \mathrm{SD}$ of three independent experiments. ${ }^{*} \mathrm{P}<0.05$, compared with the vehicle-treated control group. SBT, sea buckthorn; PQ, paraquat; NQO1, NAD(P)H dehydrogenase quinone 1; HO1, heme oxygenase 1; GPX1, glutathione peroxidase 1; GSR, glutathione reductase; $S O D 1$, superoxide dismutase 1; CAT, catalase; $P R D X 1$, peroxiredoxin 1; $L P O$, lipid perosidase; CT, control.

effect has also been observed with other cell lines $(17,21,22)$. PQ induces intracellular LDH release into the medium, which indicates a loss in cell membrane integrity. However, when the A549 cells were pretreated with SBT, the LDH level was markedly decreased. Thus, the SBT extract may block LDH release or suppress the disruption of membrane integrity by PQ. This cytoprotective effect of SBT may be due to the presence of other bioactive compounds, such as quercetin, isorhamnetin, keampferol and casuarinin in the SBT extract that may suppress the generation of intracellular ROS induced by PQ. $(10,11)$. The functional ingredients in the SBT extract have been identified and characterized. Casuarinin, one of the functional compounds in the SBT extract, has been shown to exhibit a great antioxidant effect on hydrogen peroxide-induced oxidative stress in MDCK cells (23)and an anti-inflammatory effect on $\mathrm{HaCaT}$ cells via the inhibition of $\mathrm{NF}-\kappa \mathrm{B}$ signaling (24). However, its function in the PQ-induced system has not yet been elucidated. Therefore, it is necessary to investigate the potential role of casuarinin in PQ-treated conditions. In addition, these compounds may upregulate proteins associated with the efflux transporter system, including p-glycoprotein and host defense mechanisms (such as antioxidant and detoxifying enzymes) (17). However, more studies are required to determine the mechanisms by which SBT protects the cell membrane. Total ROS was significantly increased in PQ-exposed A549 cells (Fig. 3). These results are concurrent with previous studies that investigated the effect of PQ treatment on A549 cells (16). However, the ROS level 
was gradually reduced when the cells were pretreated with different concentrations of SBT extract. Certain studies have demonstrated that SBT extract has dual effects such as ROS scavenging and antioxidant effects in in vitro and in vivo models $(9,11,21)$. Therefore, the decrease in ROS levels is due to the ROS scavenging effect or an increase in the antioxidant defense activity.

The transcription factor Nrf2 is crucial in the detoxification mechanisms for scavenging reactive metabolites and ROS. Under normal physiological conditions, inactive Nrf2 is localized in the cytoplasm by interactions with its inhibitor Keap1 (25). Under high ROS conditions, Nrf2 is released from the inhibitory complexes and translocated into the nucleus, where it regulates the expression of downstream target genes via antioxidant response elements (ARE) binding sites on their promoters (26). Previous studies have demonstrated that the stabilization and translocation of Nrf2 are critical for cytoprotection against various types of oxidative stress $(27,28)$. A lack of $N r f 2$ in MEF cells led to an increase in ROS production and apoptosis in elevated chromium (VI) and cadmium systems $(29,30)$. In the present study, SBT extract was shown to effectively induce Nrf2 gene expression in a concentration-dependent manner (Fig. 4), which significantly contributed to protection against PQ-induced cell death. Notably, Nrf2 expression was markedly induced by SBT treatment only (Fig. 4A). In addition, accumulation of Nrf2 was observed following treatment with SBT extract. Flavonoid compounds present in the SBT extract may be involved in the activation of Nrf2, as it has been shown that synthetic flavonoid compounds are potent inducers of the ARE/Nrf2/Keap1 signaling pathway (25). Several plant-derived compounds have previously been identified as inducers of phase II enzyme genes in other cellular contexts $(31,32)$. Therefore, based on these results, the effect of SBT on the expression of antioxidant-related genes, including Nrf2 target genes, such as NQO1, HOl and phase II detoxifying genes, including GPX1, GSR, SOD1, CAT, PRDX1 and LPO was determined. The SBT extract significantly induced $N Q O 1 \mathrm{mRNA}$ expression in a concentration-dependent manner but not $\mathrm{HO} 1$ mRNA expression, although both are Nrf2 target genes. It was hypothesized that the induction of $\mathrm{HOl}$ requires other transcription factors, such as $N F-\kappa B$ and $A P-1$ (33). The mechanisms underlying these differences merits further investigation, however, they are beyond the scope of the present study.

Glutathione reductase (GSR) is a pivotal enzyme of the cellular antioxidant defense mechanism, and generates the sulfhydryl form of GSH from oxidized glutathione disulfide (34). In this study, GSR expression was induced by SBT extract treatment in PQ-exposed A549 cells. This result was also supported by previous studies $(35,36)$. GPX1, which functions in the detoxification of $\mathrm{H}_{2} \mathrm{O}_{2}$, was also observed as it is the most active antioxidant enzyme in humans and has previously been associated with the antioxidant defense system $(35,36)$. In those studies, the SBT extract was shown to significantly induce GPX1 and CAT mRNA expression, which is consistent with previous studies $(10,11)$. Those results suggested that the functional SBT extract is a potent GPX1 antioxidant gene inducer and may activate the pulmonary defense system (28). Certain studies have demonstrated that SBT resists ROS stress by activating SOD1 activity and was shown to protect against butylated hydroxytoluene treatment by effective induction of SOD1 via Nrf2 activation, respectively (10). In addition, Tomita and Okuyama (37) demonstrated that LPO activity was increased in PQ intoxication. In the present study, SOD1, PRDX1 and LPO mRNA expression were not activated by pretreatment with SBT extract in A549 ells. Thus, it is likely that the Nrf2-dependent contribution of SBT extract to the induction of the expression of these antioxidant genes may be limited with regard to protecting against PQ cytoxicity. It was assumed that the non-responsiveness of SOD1, LPO and $P R D X 1$ expression to the SBT extract may be due to a lack of key compounds in the SBT extract or these may be responses that occur in the late stages rather than the early stages. However, more studies are required to understand the reason for these results. Thus, SBT may be a therapeutic candidate to protect against ROS-related diseases and cell death.

In conclusion, the data from the present study suggest that the active SBT extract exhibits potent cytoprotective effects against cell toxicity resulting from exposure to PQ via scavenging ROS, protecting the membrane integrity and inducing the expression of antioxidant-related genes, including, Nrf2, which targets divergent mechanisms. Insights were provided into the protective mechanisms of SBT under conditions that mimic PQ-poisoning with the aim of demonstrating its potential therapeutic uses for the treatment of various ROS stress-related diseases, such as PQ intoxication. At present, studies are in progress to identify specific compounds that may be used as therapeutic candidates against PQ intoxication. In addition, studies on the molecular mechanisms of SBT with regard to cell protection are currently underway.

\section{Acknowledgements}

The authors would like to thank Mrs. Tamanna Zerin for her technical support and comments. This project was supported by a grant from the Rural Development Administration, Republic of Korea (project no. PJ008246).

\section{References}

1. Schuster BG: Demonstrating the validity of natural products as anti-infective drugs. J Altern Complement Med 7 (Supp 1): S73-S82, 2001.

2. Nocentini S, Guggiari M, Rouillard D and Surgis S: Exacerbating effect of vitamin E supplementation on DNA damage induced in cultured human normal fibroblasts by UVA radiation. Photochem Photobiol 73: 370-377, 2001.

3. Kumar R, Shyam R, Divekar HM, Pahwa ML and Srivastava KK: Mechanism of increased tolerance to hypothermia after composite Indian herbal preparation II administration. J Altern Complement Med 6: 509-517, 2000.

4. Duarte J, Andriambeloson E, Diebolt M and Andriantsitohaina R: Wine polyphenols stimulate superoxide anion production to promote calcium signaling and endothelial-dependent vasodilatation. Physiol Res 53: 595-602, 2004.

5. Guliyev VB, Gul M and Yildirim A: Hippophae rhamnoides L.: chromatographic methods to determine chemical composition, use in traditional medicine and pharmacological effects. J Chromatogr B Analyt Technol Biomed Life Sci 812: 291-307, 2004.

6. Suryakumar G and Gupta A: Medicinal and therapeutic potential of sea buckthorn (Hippophae rhamnoides L.). J Ethnopharmacol 138: 268-278, 2011.

7. Kim JS, Kwon YS, Sa YJ and Kim MJ: Isolation and identification of sea buckthorn (Hippophae rhamnoides) phenolics with antioxidant activity and alpha-glucosidase inhibitory effect. J Agric Food Chem 59: 138-144, 2011 
8. Saggu S and Kumar R: Effect of seabuckthorn (Hippophae rhamnoides) leaf aqueous and ethanol extracts on avoidance learning during stressful endurance performance of rats: a dose dependent study. Phytother Res 22: 1183-1187, 2008

9. Padwad Y, Ganju L, Jain M, et al: Effect of leaf extract of Seabuckthorn on lipopolysaccharide induced inflammatory response in murine macrophages. Int Immunopharmacol 6: 46-52, 2006.

10. Maheshwari DT, Yogendra Kumar MS, Verma SK, Singh VK and Singh SN: Antioxidant and hepatoprotective activities of phenolic rich fraction of Seabuckthorn (Hippophae rhamnoides L.) leaves. Food Chem Toxicol 49: 2422-2428, 2011.

11. Bao M and Lou Y: Flavonoids from seabuckthorn protect endothelial cells (EA.hy926) from oxidized low-density lipoprotein induced injuries via regulation of LOX-1 and eNOS expression. J Cardiovasc Pharmacol 48: 834-841, 2006.

12. Kurata S, Matsumoto M, Tsuji Y and Nakajima H: Lipopolysaccharide activates transcription of the heme oxygenase gene in mouse M1 cells through oxidative activation of nuclear factor kappa B. Eur J Biochem 239: 566-571, 1996.

13. Suntres ZE: Role of antioxidants in paraquat toxicity. Toxicology 180: 65-77, 2002.

14. Bus JS and Gibson JE: Paraquat: model for oxidant-initiated toxicity. Environ Health Perspect 55: 37-46, 1984.

15. Ghio AJ: Disruption of iron homeostasis and lung disease Biochim Biophys Acta 1790: 731-739, 2009.

16. Mitsopoulos P and Suntres ZE: Cytotoxicity and gene array analysis of alveolar epithelial A549 cells exposed to paraquat. Chem Biol Interact 188: 427-436, 2010.

17. Zerin T, Kim YS, Hong SY and Song HY. Quercetin reduces oxidative damage induced by paraquat via modulating expression of antioxidant genes in A549 cells. J Appl Toxicol: Sep 20, 2012 (Epub ahead of print)

18. Geetha S, Singh V, Ram MS, Ilavazhagan G, Banerjee PK and Sawhney RC: Immunomodulatory effects of seabuckthorn (Hippophae rhamnoides L.) against chromium (VI) induced immunosuppression. Mol Cell Biochem 278: 101-109, 2005.

19. Geetha S, Sai Ram M, Singh V, Ilavazhagan G and Sawhney RC: Anti-oxidant and immunomodulatory properties of seabuckthorn (Hippophae rhamnoides) - an in vitro study. J Ethnopharmacol 79: 373-378, 2002.

20. Narayanan S, Ruma D, Gitika B, et al: Antioxidant activities of seabuckthorn (Hippophae rhamnoides) during hypoxia induced oxidative stress in glial cells. Mol Cell Biochem 278 9-14, 2005

21. Upadhyay NK, Kumar R, Mandotra SK, et al: Safety and healing efficacy of Sea buckthorn (Hippophae rhamnoides L.) seed oil on burn wounds in rats. Food Chem Toxicol 47: 1146-1153, 2009.

22. Ganju L, Padwad Y, Singh R, et al: Anti-inflammatory activity of seabuckthorn (Hippophae rhamnoides) leaves. Int Immunopharmacol 5: 1675-1684, 2005.

23. Chen CH, Liu TZ, Kuo TC, et al: Casuarinin protects cultured MDCK cells from hydrogen peroxide-induced oxidative stress and DNA oxidative damage. Planta Med 70: 1022-1026, 2004.
24. Kwon DJ, Bae YS, Ju SM, Goh AR, Choi SY and Park J: Casuarinin suppresses TNF-alpha-induced ICAM-1 expression via blockade of $\mathrm{NF}-\kappa \mathrm{B}$ activation in $\mathrm{HaCaT}$ cells. Biochem Biophys Res Commun 409: 780-785, 2011.

25. Zerin T, Kim YS, Hong SY and Song HY: Protective effect of methylprednisolone on paraquat-induced A549 cell cytotoxicity via induction of efflux transporter, P-glycoprotein expression. Toxicol Lett 208: 101-107, 2012.

26. Surh YJ, Kundu JK and Na HK: Nrf2 as a master redox switch in turning on the cellular signaling involved in the induction of cytoprotective genes by some chemopreventive phytochemicals. Planta Med 74: 1526-1539, 2008.

27. Jaiswal AK: Nrf2 signaling in coordinated activation of antioxidant gene expression. Free Radic Biol Med 36: 1199-1207, 2004.

28. Kode A, Rajendrasozhan S, Caito S, Yang SR, Megson IL and Rahman I: Resveratrol induces glutathione synthesis by activation of Nrf2 and protects against cigarette smoke-mediated oxidative stress in human lung epithelial cells. Am J Physiol Lung Cell Mol Physiol 294: L478-L488, 2008.

29. Cho HY, Jedlicka AE, Reddy SP, et al: Role of NRF2 in protection against hyperoxic lung injury in mice. Am J Respir Cell Mol Biol 26: 175-182, 2002.

30. He X, Lin GX, Chen MG, Zhang JX and Ma Q: Protection against chromium (VI)-induced oxidative stress and apoptosis by Nrf2. Recruiting Nrf2 into the nucleus and disrupting the nuclear Nrf2/Keap1 association. Toxicol Sci 98: 298-309, 2007.

31. Chen J and Shaikh ZA: Activation of Nrf2 by cadmium and its role in protection against cadmium-induced apoptosis in rat kidney cells. Toxicol Appl Pharmacol 241: 81-89, 2009.

32. Singh M, Murthy V and Ramassamy C: Standardized extracts of Bacopa monniera protect against $\mathrm{MPP}^{+}$- and paraquat-induced toxicity by modulating mitochondrial activities, proteasomal functions, and redox pathways. Toxicol Sci 125: 219-232, 2012.

33. Liu XP, Goldring CE, Copple IM, et al: Extract of Ginkgo biloba induces phase 2 genes through Keap1-Nrf2-ARE signaling pathway. Life Sci 80: 1586-1591, 2007.

34. Camhi SL, Alam J, Wiegand GW, Chin BY and Choi AM: Transcriptional activation of the HO-1 gene by lipopolysaccharide is mediated by $5^{\prime}$ distal enhancers: role of reactive oxygen intermediates and AP-1. Am J Respir Cell Mol Biol 18: 226-234, 1998.

35. Fujii J, Ito JI, Zhang $X$ and Kurahashi T: Unveiling the roles of the glutathione redox system in vivo by analyzing genetically modified mice. J Clin Biochem Nutr 49: 70-78, 2011.

36. Singh A, Boldin-Adamsky S, Thimmulappa RK, et al: RNAi-mediated silencing of nuclear factor erythroid-2-related factor 2 gene expression in non-small cell lung cancer inhibits tumor growth and increases efficacy of chemotherapy. Cancer Res 68: 7975-7984, 2008

37. Rangasamy T, Cho CY, Thimmulappa RK, et al: Genetic ablation of Nrf2 enhances susceptibility to cigarette smoke-induced emphysema in mice. J Clin Invest 114: 1248-1259, 2004.

38. Tomita M and Okuyama T: Effect of paraquat on the malondialdehyde level in rat liver microsomes (in vitro). Arch Toxicol 68: 187-192, 1994 